\title{
Mango de azúcar (Mangifera indica), variedad de Colombia: características antioxidantes, nutricionales y sensoriales
}

\author{
Sugar mango (Mangifera indica), \\ variety from Colombia: antioxidant, \\ nutritional and sensorial characteristics
}

\begin{abstract}
The sugar mango is a small Colombian cultivar with low fiber content, good aroma and flavor. This research evaluated antioxidant capacity (FRAP, DPPH) of the fruit at different ripening stages classified by visual inspection and CIELab scale. Nutritional and sensory characteristics and ORAC value were measured in the ripe fruit. Sensorial characteristics of two mango nectars sweetened with sucrose and sucralose using ripe fruit were determined by affective acceptance test and Hedonic scale. Results showed that classification by visual inspection coincided with the CIELab scale for all ripening stages. The green mango has 45\% more flavonoids than the mature one. The carotenoid content, DPPH and FRAP values were similar in the different ripening stages. Sucralose sweetened nectar was accepted by $94.9 \%$ compared with that sweetened with sucrose $(89.1 \%)$. Results indicate that mango sugar fruit and nectar present antioxidant capacity, bioactive and nutritional compounds with potential health benefits. Key words: nectar; Mangifera indica; antioxidant capacity; sensory.
\end{abstract}

Andrea Corrales-Bernal (1)

Maria Elena Maldonado (1)

Luz Amparo Urango (1)

Maria Camila Franco (1)

Benjamín Alberto Rojano (2)

(1) Grupo Impacto de los Componentes Alimentarios en la Salud, Escuela Nutrición y Dietética, Universidad de Antioquia, Medellín, Colombia. (2) Grupo de Ciencia de los Alimentos, Facultad de Ciencias, Universidad Nacional de Colombia, sede Medellín, Colombia.

$$
\begin{array}{r}
\text { Dirigir correspondencia a: } \\
\text { sra. } \\
\text { Maria Elena Maldonado C. } \\
\text { Ciudadela Robledo Bloque 44 } \\
\text { Universidad de Antioquia } \\
\text { Carrera } 75 \$ 67-87 \\
\text { Medellín, Colombia } \\
\text { Fax: }(+57-4) 2305007 \\
\text { Teléfono (+57-4) } 2199223 \\
\text { E-mail: maria.maldonado@udea.edu.co }
\end{array}
$$

Este trabajo fue recibido el 10 de Abril de 2014 y aceptado para ser publicado el 7 de Agosto de 2014.

\section{INTRODUCCIÓN}

El mango (Mangifera indica) es un alimento fuente de ácido ascórbico (9.79 a 186 mg /100 g), carotenoides (1159 a 3000 mg/100 g) y (poli)fenoles presentes en la parte comestible de la fruta que le confieren capacidad antioxidante (1-10). Es una de las frutas tropicales más importantes del mundo en términos de producción, superficie cultivada, y popularidad, con una producción global que excede los 27 millones de toneladas, siendo el mayor cultivo tropical seguido por el banano, la piña, papaya y aguacate (11).

La calidad nutracéutica del mango ha sido estudiada en función de su capacidad antioxidante, el contenido de (poli) fenoles y carotenoides totales en variedades como Francis, Kent, Ataulfo, Tommy Atkins, Haden (3, 5, 6, 12-16). Adicionalmente, en la mayoría de estos trabajos se ha utilizado las variedades de Brasil $(1,6)$, Estados Unidos (13), China (5, 14), India (15,17), Cuba (16), Tailandia (18), España (19). Sin embargo, no existen estudios sobre las característcias químicas, nutricionales, sensoriales y antioxidantes de variedades de Colombia, vigésimo productor de mango en el mundo según la Food Agriculture Organization (FAO), con un aporte de 239 mil toneladas, equivalente al $0.6 \%$ de la producción mundial (20).

En Colombia, 39\% del área ocupada por esta fruta corresponde a plantaciones silvestres de mango variedad hilacha, con cero tecnificación y carencia absoluta de asistencia técnica; le siguen las variedades Tommy Atkins (20\%), Keitt (11\%) y con $5 \%$ el mango de azúcar (20); ésta última cultivada principalmente en la región norte de Colombia, cerca de la Costa Atlántica, cuyo cultivo se ha extendido a los departamentos de Cundinamarca, Tolima y Huila. El mango de azúcar presenta una alta demanda como fruta fresca en el exterior por su tamaño pequeño, escasa fibra, buen aroma y sabor; además desde el punto de vista comercial es la variedad más rentable pues tiene casi diez meses de buen precio en comparación con las anteriores (21).

Esta investigación consistió en caracterizar la capacidad antioxidante y el contenido de compuestos bioactivos presentes en diferentes estadios de maduración del mango de azúcar. Además, se analizó la composición proximal, la capacidad 
antioxidante y el perfil sensorial de una bebida tipo néctar realizada con mango de azúcar maduro. Los hallazgos del presente estudio pretenden apoyar la importancia del consumo de frutas, particularmente del mango en Colombia, donde $8,7 \%$ de la población lo consume con una ingesta promedio por día de 147,7 g de mango (22). Adicionalmente, mostrar que la variedad colombiana mango de azúcar presenta características diferentes a las de otras variedades bien estudiadas, y que son factores importantes para su aprovechamiento nutricional y para la elaboración de productos con potencial beneficio para la salud.

\section{MATERIAL Y MÉTODOS}

Material vegetal y preparación del néctar

Las frutas utilizadas pertenecen a la variedad mango de azúcar proveniente de la costa Caribe colombiana. Se seleccionaron las frutas maduras por inspección visual como verdes, pintonas y maduras según la Norma Técnica Colombiana (NTC) 5139 (23); luego se obtuvo las coordenadas según el sistema RGB (Red, Green,Blue) mediante colorímetro Color Analyzer Probe LT Lutron RGB-1002, estos valores fueron convertidos a escala CIELAB. Las frutas seleccionadas se lavaron, desinfectaron (hipoclorito de sodio 100ppm) y despulparon para ser congeladas. El néctar se preparó descongelando la pulpa en refrigerador $\left(4^{\circ} \mathrm{C}\right)$ toda la noche, diluida en agua destilada ( 1 pulpa: 4 agua), endulzada con sucralosa o sacarosa hasta alcanzar $13^{\circ}$ Brix, estabilizante (gelatina sin sabor $0.07 \%$ ). Los ingredientes fueron mezclados en una licuadora industrial, pasteurizados $\left(90^{\circ} \mathrm{C}, 3 \mathrm{~min}\right)$, y envasados en condiciones asépticas y almacenadas a 4 ${ }^{\circ} \mathrm{C}$, protegido de la luz. Se evaluó la calidad microbiológica de la pulpa y del néctar mediante conteo total de bacterias mesofílicas aerobias, hongos y levaduras, cuenta de Coliformes totales y fecales, Escherichia coli según NTC 4458 de 1998 (24); y Salmonella sp, para evaluar Salmonella según NTC 4574 (25); y no se obtuvo crecimiento microbiano en el producto final. Posteriormente, se analizó humedad (pérdida por secado) según la Guía Técnica Colombiana 1.14, cenizas por el método directo - Association of Official Agricultural Chemists (AOAC) 923.03, el nitrógeno total y la proteína total (coeficiente 6,25) por el método Microkjeldhal - AOAC 954.01, los carbohidratos totales y las calorías se calcularon a partir de los componentes (26).

FRAP (Ferric reducing antioxidant power)

Mide capacidad reductora de una muestra mediante el aumento de la absorbancia por la formación del complejo 2,4,6-tri(2-piridil)-s-triazina (TPTZ) a su forma ferrosa $\left(\mathrm{Fe}^{+2}\right)$ a $595 \mathrm{~nm}$ (27). Los resultados se reportaron como $\mathrm{mg}$ de ácido ascórbico (AA)/100g de pulpa.

\section{DPPH (2,2-diphenylpicrylhydrazyl)}

Mide la capacidad de la muestra para neutralizar el radical DPPH por transferencia de hidrógeno a absorbancia a $517 \mathrm{~nm}$. Los resultados se reportaron como valores $\mu \mathrm{mol}$ equivalentes de trolox/ 100g pulpa (TEAC) (28).

\section{ORAC (Oxygen Radical Absorbance Capacity)}

Se midió la actividad antioxidante del extracto contra el radical peroxilo 2,2 -Azo-bis (2-amidinopropano) dihydrochlorido (AAPH) generado a $37^{\circ} \mathrm{C}$ (29). La fluoresceína se usó como sonda cuya disminución indica la cantidad del radical peroxilo inhibido. Los resultados se expresaron como TEAC respecto a una curva de calibración de Trolox.

\section{Determinación de fenoles totales}

Se realizó por el método colorimétrico de Folin-Ciocalteau (30). A una porción de pulpa de mango (1 g) se le adicionó agua destilada, reactivo de Folin-Ciocalteu y una solución de bicarbonato de sodio al $7.1 \%$. Las lecturas se realizaron a una longitud de onda de $760 \mathrm{~nm}$ en un espectrofotómetro Genesys 20. Se construyó una curva patrón usando como estándar ácido gálico y los resultados se expresaron como equivalentes en $\mathrm{mg}$ de ácido gálico/100 g de pulpa (GAE).

\section{Determinación de flavonoides}

Se empleó el ensayo colorimétrico con cloruro de aluminio usado por Debnath en 2011 (31). A una pórción de pulpa de mango (1 g) se le adicionó agua destilada, solución de nitrato de sodio al $5 \%(\mathrm{v} / \mathrm{v})$. La mezcla se incubó por 5 minutos. Posteriormente, 10\% (v/v) de solución de cloruro de aluminio fue mezclado con la mezcla anterior. Se construyó una curva patrón usando como estándar catequina. La absorbancia se leyó a $510 \mathrm{~nm}$ y el contenido de flavonoides se expresó como mg catequina/100g de pulpa.

\section{Determinación de carotenoides}

Se usó el método descrito por Biswas et al en 2011 (32). Se tomó en un tubo de ensayo un $1 \mathrm{~g}$ de muestra, se adicionaron $5 \mathrm{~mL}$ de acetona fría se dejó reposar alrededor de $15 \mathrm{~min}$ en refrigerador $\left(4^{\circ} \mathrm{C}\right)$, pasado este tiempo se agitó en vórtex por $2 \mathrm{~min}$. El precipitado se re-extrajo con $5 \mathrm{~mL}$ de acetona fría y se repitió todo el proceso anterior. Ambos extractos acetónicos se mezclaron, luego se filtraron en papel Whatmann No. 42 y se determinó la absorbancia en un espectrofotómetro UV-Vis Thermo Scientific Genesys 20® a longitud de onda de 449 $\mathrm{nm}$. La concentración de carotenoides se estipuló mediante la curva de calibración respectiva, utilizando $\beta$-Caroteno como sustancia patrón. Los resultados se expresaron como $\mathrm{mg} \beta$-Caroteno/100 g pulpa.

Prueba de análisis sensorial de los néctares

Se utilizó el método afectivo por prueba de nivel de agrado con escala hedónica de 3 puntos: "me gusta", "ni me gusta ni me disgusta" y "me disgusta", para color, olor, sabor, dulzor y la consistencia (33); y una prueba de aceptación de las muestras. El producto fue evaluado por 429 panelistas no entrenados de ambos géneros (masculino y femenino).

\section{ANÁLISIS ESTADÍSTICO}

Los resultados se analizaron con SAS versión 9.0. Los datos se expresaron como promedio \pm error estándar de la media (ESM). Las diferencias estadísticas se evaluaron con ANOVA de una vía y post-test de comparaciones múltiples Tukey con base a un nivel de significancia de 5\%. El análisis de las pruebas sensoriales se realizó por medio de la técnica multivariada de la varianza MANOVA, mediante el modelo lineal general (GLM), cuyo objetivo fue comparar el efecto de los tratamientos (néctar con sacarosa y néctar con sucralosa) y del género (masculino y femenino), teniendo en cuenta de manera simultánea las variables respuesta color, olor, sabor, dulzor y consistencia. Se utilizó contraste canónico de tipo ortogonal determinación vía máxima verosimilitud la dimensionalidad de contraste; se utilizó el paquete estadístico.

\section{RESULTADOS}

Clasificación de la fruta, características antioxidantes y metabolitos

La inspección visual permitió hacer una clasificación de 
la fruta en los tres estados de maduración (NTC 5139). Los parámetros $\mathrm{a}^{*} \mathrm{y} \mathrm{b}^{*}$ fueron significativamente diferentes entre los tres estadíos de maduración del mango, pero no el parámetro L (tabla 1). La inspección visual coincidió con los valores de la escala CIELab, particularmente con el parámetro a* que comprende desde el color verde (valores negativos) hasta el color rojo (valores positivos) (tabla 1). La capacidad antioxidante (DPPH) y reductora (FRAP) de la pulpa de mango de azúcar fue significativamente diferente y mayor para el mango pintón con respecto a su estado verde y maduro (Tabla 2). Con relación al contenido de metabolitos secundarios, la pulpa de mango verde contiene $45 \%$ más flavonoides que la pulpa madura, siendo un valor significativamente mayor. Mientras que el contenido de carotenoides totales fue significativamente diferente entre la pulpa pintona y madura (tabla 3). El valor ORAC (hidrofílico: 2209,2 \pm 25,2 y lipofílico: 442,4 $\pm 29,7$
TEAC) y el contenido de fenoles totales $(217,6+1,4$ GAE)se determinaron solo para la madura, con la cual se elaboraron las formulaciones mencionadas en materiales y métodos.

Análisis proximal y sensorial del néctar

El análisis proximal de la pulpa de mango de azúcar en estado maduro posee un alto contenido de humedad y poco contenido de grasa total, carbohidratos totales, cenizas y calorías (tabla 4). El contenido total de sólidos solubles fue de $6^{\circ}$ a $13^{\circ}$ Brix para el néctar con sucralosa y sacarosa, respectivamente. El contenido total de sólidos solubles en las dos formulaciones varió según el tipo de endulzante, por lo que se necesitó poca cantidad de endulzante no calóricos para obtener el grado de dulzor similar al del azúcar, y para no aumentar la cantidad de sólidos solubles en el néctar. Al efectuar el análisis multivariado de la varianza no se observó

\section{TABLA 1}

Parámetros de color según estado de maduración del mango de azúcar.

\begin{tabular}{|c|c|c|c|}
\hline Estado de maduración & $\mathrm{L}$ & $\begin{array}{c}\text { Parámetros } \\
\text { a }\end{array}$ & $b$ \\
\hline Mango verde & $21,3 \pm 0,3$ & $-4,0 \pm 0,3^{a}$ & $10,4 \pm 0,3^{a}$ \\
\hline Mango pintón & $20,4 \pm 0,9$ & $4,1 \pm 0,3^{b}$ & $13,0 \pm 0,3^{b}$ \\
\hline Mango maduro & $22,0 \pm 0,6$ & $17,3 \pm 0,9^{c}$ & $19,0 \pm 0,2^{c}$ \\
\hline
\end{tabular}

Promedio \pm Error estándar de la media, $(n=3)$.

Los valores dentro de cada parámetro que no comparten la misma letra, son significativamente diferentes: $a \neq b \neq c, P<0,05$.

TABLA 2

Capacidad antioxidante de la pulpa del mango de azúcar.

\begin{tabular}{lcc}
\hline Estado de maduración & DPPH & $\begin{array}{c}\text { FRAP } \\
\text { (mg AA/100 g pulpa) }\end{array}$ \\
Mango verde & (TEAC/100 g pulpa) & $480,1+6,2^{\mathrm{a}}$ \\
Mango pintón & $5523,6+221,9^{\mathrm{a}}$ & $525,5+13,8^{\mathrm{b}}$ \\
Mango maduro & $6471,5+167,7^{\mathrm{b}}$ & $508,4+27,1^{\mathrm{a}, \mathrm{b}}$ \\
\hline
\end{tabular}

Promedio + error estándar de la media, $(n=4)$.

Los valores dentro de cada tipo de prueba antioxidante que no comparten la misma letra, son significativamente diferentes: $a \neq b, P<0,05$.

\section{TABLA 3}

Metabolitos de la pulpa del mango de azúcar.

Estado de maduración

Mango verde
Mango pintón
Mango maduro
Flavonoides (mg catequina/100 g pulpa)

$$
\begin{aligned}
& 81,6+3,6^{a} \\
& 71,8+2,2^{b} \\
& 56,2+4,4^{b}
\end{aligned}
$$

\author{
Carotenoides \\ (mg bcaroteno/100 g pulpa)
}

$$
\begin{gathered}
11,1+0,01^{a, b} \\
11,8+0,2^{a} \\
10,7+0,2^{b}
\end{gathered}
$$

Promedio + error estándar de la media, $(n=4)$.

Los valores dentro de cada tipo de prueba antioxidante que no comparten la misma letra, son significativamente diferentes: $\mathrm{a} \neq \mathrm{b}, \mathrm{P}<0,05$. 
diferencia estadística entre los tratamientos, es decir, entre el néctar endulzado comparado con el de sucralosa, ni tampoco se encontró diferencia entre géneros (tabla 5).

Para la variable de aceptación se encontró diferencia entre los tratamientos $(p<0.05)$ donde el néctar con sacarosa azúcar fue el de mayor aceptación, resaltando que entre géneros no se detectó diferencia estadística $(p>0.05)$ (tabla 6). Con relación al análisis sensorial se obtuvo que la mayoría de los consumidores manifestaron gustarles todas las variables evaluadas en ambas formulaciones de néctar. La mayor aceptación fue para el néctar elaborado con sucralosa (94,9\%) (Figura 1B) comparado con el néctar que contenía sacarosa (89,1\%) (Figura 1A) según el análisis descriptivo.

\section{DISCUSIÓN}

En el presente estudio se describe por primera vez la capacidad antioxidante, el contenido de flavonoides y carotenoides del mango de azúcar en diferentes estados de ma- duración. Se presenta la composición en macronutrientes de la pulpa de la fruta madura, con la cual se formuló un néctar con dos tipos de endulzantes, sacarosa y sucralosa, sometido a evaluación sensorial.

La caracterización del mango de azúcar se inició con la clasificación por color (parámetros $L, a, b$ ), considerado índice de calidad en las frutas. Los valores $L$ y $b$ indican claridad y color amarillo respectivamente.

El parámetro L se usa como indicador de pardeamiento de la superficie en respuesta a la acción de enzimas como la polifenol oxidasa y peroxidasa de la piel o pulpa (34). El valor del parámetro L fue similar en los tres estados de maduración del mango de azúcar, lo que indica que al menos en la piel no se evidenció pardeamiento.

El valor del parámetro b fue mayor en el estado maduro, indicando mayor coloración amarilla que los anteriores, coherente con el parámetro a que evidenció cambios de color desde el verde (mango en estado verde) hasta el rojo (mango

TABLA 4

Análisis bromatológico de la pulpa madura de mango de azúcar (Mangifera indica L.) /100 g

\begin{tabular}{lc}
\hline Parámetros & Valores obtenidos \\
& $\%$ \\
Humedad & 79.3 \\
Ceniza & 0.4 \\
Grasa total & 0.02 \\
Nitrógeno total & 0 \\
Proteína Total & 0 \\
Carbohidratos totales & 20.3 \\
Calorías Kilocalorías/100 g & 81.5 \\
\hline
\end{tabular}

TABLA 5

Análisis multivariado entre los néctares y entre género.

\begin{tabular}{lcc}
\hline Prueba estadística & Tratamientos & Género \\
& $\operatorname{Pr}>\mathrm{F}$ & $\mathrm{F}$ \\
Wilks' Lambda & 0,35 & 0,44 \\
Pillai's Trace & 0,35 & 0,44 \\
Hotelling-Lawley Trace & 0,35 & 0,44 \\
Roy's Greatest Root & 0,35 & 0,44 \\
\hline
\end{tabular}

\section{TABLA 6}

Análisis multivariado para la variable aceptación.

Fuente de variación

Tratamientos

Género
Pr $>$ F

0.029

0.25 
en estado maduro). Adicionalmente, el blanqueamiento y pasteurización de la pulpa de mango de azúcar madura pudo haber inactivado estas enzimas (35), permitiendo conservar estos parámetros en las dos formulaciones de néctar elaborado.

El análisis bromatológico en la pulpa del mango de azúcar no difiere de los realizados por Stafford en cuanto a la humedad $(81,7 \%)$ y cenizas $(0,39 \%)$; pero si en el contenido de carbohidratos $(16,8 \%)$, grasas $(0,4 \%)$, proteínas $(0,7 \%)$ y aporte calórico (66 Kcal) (36).

En diferentes variedades de mango se ha reportado el contenido de flavonoides y carotenoides totales, compuestos químicos que actúan como antioxidantes. El mango Ataulfo es uno de los que posee un alto contenido de flavonoides totales en la pulpa (125 mg catequina/100g pulpa) asociado con su larga vida útil (37). El contenido de flavonoides del mango de azúcar maduro disminuyó con su maduración y fue menor al reportado para la variedad Ataulfo, lo que podría favorecer reacciones de deterioro en el tiempo para el mango de azúcar que reducen su tiempo de vida útil.

Con relación al contenido de carotenoides totales en el mango de azúcar,aunque se presentó variación significativa entre los estadíos pintón y maduro, los valores obtenidos en los tres grados de maduración analizados fueron similares a los valores reportados para las variedades Kent y Keitt $(12,9$ y 10,4 mg ßcaroteno/100 g pulpa, respectivamente); mayores al Tommy Atkins, Tainong N¹, Irwin, JinHwang $(4,9 ; 5,2 ; 3.7$; 2,6 respectivamente) (14); pero menores al mango Ataulfo (26.1) (2)

En cuanto al contenido de fenoles totales en la fruta madura, este fue alto considerando que la Corporación Colombiana de Investigación Agropecuaria (CORPOICA) el año 2008 reportó un contenido entre 50 y 200 mg GAE/100g en el mango criollo colombiano (12). Incluso fue mayor al rango reportado de fenoles totales de las variedades Tommy Atkins, Kent, Keitt y Haden (31.2 mg GAE/100 g pulpa), Ataulfo y Ubá (109.3 y 208,7 mg GAE/100 g pulpa, respectivamente) $(2,19)$.

La determinación del contenido total de fenoles se realizó con el método Folin-Ciocalteau, el cual no es recomendable utilizar en frutas con alto contenido de ácido ascórbico como el mango, porque éste último contribuye a reducir los azúcares, las proteínas solubles y otras sustancias que pueden sobreestimar el contenido de fenoles totales en el mango (30). Sin embargo, se ha descrito que los principales compuestos fenólicos presentes en el mango son el ácido gálico y la quercetina (19). A pesar de las desventajas del método Folin-Ciocalteau, éste método se utilizó aquí para una primera caracterización nunca antes hecha del mango de azúcar. Actualmente nuestro grupo se encuentra realizando la determinación de compuestos

\section{FIGURA 1}

Prueba de aceptación del néctar de mango de azúcar elaborado con sacarosa (A) y sucralosa (B).

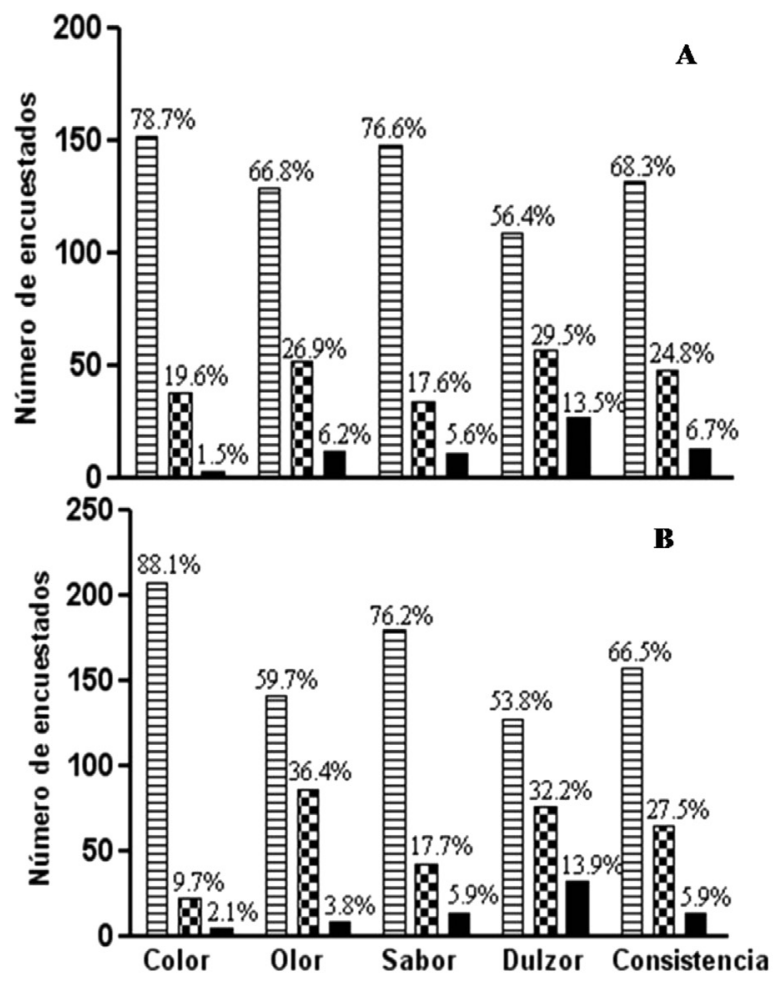

目 Me gusta $\mathbf{X}$ Ni me gusta ni me disgusta $\square$ No me gusta 
fenólicos en la pulpa de esta fruta mediante otras estrategias metodológicas como la cromatografía líquida de alta presión (HPLC).

El valor DPPH en la pulpa de mango de azúcar en los tres estadios fue mayor al valor promedio reportado para las variedades criollas colombianas (5000 TEAC) (12) y las variedades chinas Jinhuang, Tainong 1, Guifei, Ao, Xiaoji, Fengshunwuhe, Mallika e Irwin (461-2.930 $\mu \mathrm{mol}$ trolox/100g) en estado maduro $(5,14)$. Por el contrario, el valor FRAP del mango de azúcar en los tres estados de maduración resultó inferior al reportado para las variedades chinas antes mencionadas $(910-6.769$ TEAC) $(5,14)$.

Los valores DPPH y FRAP aquí obtenidos indican que la pulpa de mango de azúcar contiene sustancias con capacidad para atrapar radicales libres y para reducir el ion $\mathrm{Fe}^{3+}$ a $\mathrm{Fe}^{2+}$, respectivamente, las cuales podrían ser ácido ascórbico, iones metálicos (calcio: 10 mg, Hierro: 0,4 mg / 100 gr pulpa) (36) y fenoles reductores.

Con relación al valor ORAC, la prueba más utilizada para valoración antioxidante de alimentos de consumo habitual por los humanos (38), indica la capacidad de antioxidantes hidrofílicos tales como el ácido ascórbico y compuestos fenólicos, así como la capacidad antioxidante de compuestos hidrofílicos como carotenoides presentes en la pulpa madura de mango de azúcar. El valor ORAC ( $\mu$ mol trolox/100g pulpa) total para el mango de azúcar resultó ser mayor al de las variedades Ataulfo (326 - 931), Haden (225), Francis (219), Kent (150) y Tommy Atkins (156); pero menor al rango promedio reportado para las variedades chinas aquí mencionadas (3248 - 8545) $(2,5,14)$.

Finalmente, la evaluación sensorial nos mostró que una bebida tipo néctar a base de mango de azúcar (maduro) libre de conservantes, acidulantes, colorantes artificial y endulzado con sacarosa o sucralosa, es una buena alternativa para fomentar el consumo de esta fruta. Mientras que las bebidas comerciales de néctar de mango contienen 22 a 37,9 g de azúcares, 0 a $1,8 \mathrm{~g}$ de fibra, 88 a 145 calorías y no aportan micronutrientes como hierro, calcio, vitamina A y C. Particularmente, en nuestra preparación, la adición del estabilizante ni de sucralosa alteró las características sensoriales; y la concentración de pulpa empleada podría aportar los componentes bioactivos con carácter antioxidante antes mencionados.

\section{CONCLUSIONES}

El mango de azúcar es una fruta con un contenido importante en fenoles totales y carotenoides, especialmente en la pulpa madura que le confieren propiedad antioxidante comparable, y en algunos casos mayor a otras variedades de mango más estudiadas y con mayor valor comercial que la fruta aquí descrita.

Estos hallazgos pretenden beneficiar a toda su cadena productiva, al consumidor final y apoyar la importancia del consumo de frutas en la dieta humana.

Nuestro grupo continúa con la descripción en el contenido nutricional, de fenoles, flavonoides y carotenoides presentes en la pulpa maduraa, y el estudio de la vida útil de este producto en función de sus características antioxidantes, sensoriales y compuestos bioactivos como valor agregado.

\section{RESUMEN}

El mango de azúcar es una variedad colombiana pequeña, de escasa fibra, buen aroma y sabor. Esta investigación evaluó la capacidad antioxidante del fruto en diferentes estados de maduración por FRAP y DPPH clasificados por inspección visual y escala CIELab. Las características nutricionales y el valor ORAC se determinaron en el mango maduro. Las características sensoriales de dos formulaciones (sacarosa y sucralosa) con mango maduro se determinaron por prueba afectiva de aceptación y escala Hedónica. Resultados: la clasificación por inspección visual coincidió con la escala CIELab. El mango verde tiene $45 \%$ más flavonoides que el maduro. El contenido de carotenoides, valores DPPH y FRAP fueron similares en los diferentes estados de maduración. El 94\% de los panelistas aceptaron el néctar con sucralosa comparado con el endulzado con sacarosa (89,1\%). Los resultados indican que el mango de azúcar y un producto tipo néctar poseen capacidad antioxidante, compuestos bioactivos y nutricionales beneficiosos para la salud.

Palabras clave: néctar; Mangifera indica; capacidad antioxidante; sensorial.

Agradecimientos: Este trabajo fue financiado por el Comité para el Desarrollo de la Investigación (CODI) de la Universidad de Antioquia, código 624/convocatoria de mediana cuantía 2011. Agradecemos al bioterio de la Corporación para Investigaciones Biológicas (CIB), al laboratorio de Alimentos Funcionales de la Universidad Nacional de Colombia sede Medellín y a COLCIENCIAS por la beca otorgada a la estudiante de doctorado.

\section{BIBLIOGRAFÍA}

1. Valente A, Albuquerque TG, Sanches-Silva A, Costa HS. Ascorbic acid content in exotic fruits: $A$ contribution to produce quality data for food composition databases. Food Res Int. 2011; 44: 2237-42.

2. Manthey JA, Perkins-Veazie P. Influences of harvest date and location on the levels of beta-carotene, ascorbic acid, total phenols, in vitro antioxidant capacity, and phenolic profiles of five commercial varieties of mango (Mangifera indica L.). J Agricult Food Chem. 2009; 57:10825-30.

3. Godoy HT, Rodriguez-Amaya DB. Carotenoid composition of commercial mangoes from Brazil. LWT Food Sci Technol. 1989; 22: 100-103.

4. Kim Y, Brecht JK, Talcott ST. Antioxidant phytochemical and fruit quality changes in mango (Mangifera indica L.) following hot water immersion and controlled atmosphere storage. Food Chem. 2007; 105: 1327-34.

5. Ma X, Wu H, Liu L, Yao Q, Wang S, Zhan R, Xing S, Zhou $Y$. Polyphenolic compounds and antioxidant properties in mango fruits. Scientia Horticulturae 2011; 129: 102-7.

6. Rocha Ribeiro SM, Queiroz JH, Lopes Ribeiro de Queiroz ME, Campos FM, Pinheiro Sant'ana HM. Antioxidant in mango (Mangifera indica L.) pulp. Plant Foods Hum Nutr. 2007; 62: 13-7.

7. Vasco C, Ruales J, Kamal-Eldin A. Total phenolic compounds and antioxidant capacities of major fruits from Ecuador. Food Chem. 2008. 111: 816-823.

8. Dembitsky VM, Poovarodom S, Leontowicz H, Leontowicz M, Vearasilp S, Trakhtenberg S, Gorinstein S. The multiple nutrition properties of some exotic fruits: Biological activity and active metabolites. Food Res Int. 2011; 44: 1671-701.

9. Martínez $R$, Torres $P$, Meneses MA, Figueroa JG, PérezÁlvarez JA, Viuda-Martos M. Chemical in vitro antioxidant properties of mango, guava, pineapple and passion fruit dietary fiber concentrate. Food Chem. 2012; 135:1520-6.

10. Belloso O, Gorinstein S, González-Aguilar GA. Quality index, consumer acceptability, bioactive compounds, and antioxidant activity of fresh-cut "ataulfo" mangoes (man- 
gifera indica L.) as affected by low-temperature storage. J Food Sci. 2009; 74: S126-S34.

11. FAOSTAT. 2008. FAO Statistical Databases Agriculture. Available from: http://faostat.fao.org. Accessed Nov 2012.

12. García J, Floriano JA, Corredor JP, Bernal JA, Vásquez $L A$, Sandoval AP. Atributos de calidad del mango criollo para la agroindustria, CORPOICA ed. Bogotá, D.C., Produmedios. p. 72, 2008.

13. Noratto GD, Bertoldi MC, Krenek K, Talcott ST, Stringheta PC, Mertens-Talcott SU. Anticarcinogenic effects of polyphenolics from mango (Mangifera indica) varieties. J Agric Food Chem. 2010; 58: 4104-12.

14. Liu F-X, Fu X-F, Chen F, Liao X-J, Hu X-S, Wu J-H. Physicochemical and antioxidant properties of four mango (Mangifera indica L.) cultivars in China. Food Chem. 2013; 138: 396-405.

15. Pandit SS, Chidley HG, Kulkarni RS, Pujari KH, Giri AP, Gupta VS. Cultivar relationships in mango based on fruit volatile profiles. Food Chem. 2009; 114: 363-72.

16. Pino JA, Mesa J, Muñoz Y, Martí P, M, Marbot R. Volatile components from mango (Mangifera indica L.) cultivars. J Agric Food Chem. 2009; 53: 2211-23.

17. Sha SK, Sethi S, Srivastav M, Dubey AK, Sharma RR, Samuel $D V K$, Singh AK. Firmness characteristics of mango hybrids under ambient storage. J Food Eng. 2010; 97: 208-12.

18. Ledeker CN, Suwonsichon S, Chambers DH, Adhikari K. Comparison of sensory attributes in fresh mangoes and heat-treated mango purées prepared from Thai cultivars. LWT-Fodd Sci Technol. 2014; 56:138-44.

19. Robles-Sánchez RM, Islas-Osuna MA, Astiazarán-García $H$, Vázquez-Ortiz FA, Martín-Belloso O, Gorinstein S, González-Aguilar GA. Quality index, consumer acceptability, bioactive compounds, and antioxidant activity of fresh-cut "ataulfo" mangoes (mangifera indica L.) as affected by low-temperature storage. J Food Sci. 2009; 74: S126-S34.

20. Alvarado-Moreno JR. Las tareas del acuerdo de competitividad de la cadena del mango. Rev Frutas Hortalizas 2012; 25: 16- 19.

21. Corpoica. [Consultado 2014 Mar 25]. Disponible en: http://www.corpoica.org.co/sitioweb/Noticias/vernoticia. asp?id_noticia $=888$

22. Instituto Colombiano de Bienestar Familiar. Encuesta Nacional de la Situación Nutricional de Colombia (ENSIN) 2005. Bogotá, D.C., El Instituto, 2006.

23. Instituto Colombiano de Normas Técnicas y Certificación ICONTEC. Norma técnica Colombiana 5139. Frutas frescas. Mangos criollos. Especificaciones. Bogotá, D.C, El Instituto, 2004.

24. Instituto Colombiano de Normas Técnicas y Certificación ICONTEC. Norma técnica Colombiana 4458. Microbiología de alimentos y de alimentos para animales. Método horizontal para el recuento de coliformes o Escherichia cole o ambos. Técnica de recuento de colonias utilizando medios fluorogénicos o cromogénico. Bogotá, D.C, El Instituto, 2007.

25. Instituto Colombiano de Normas Técnicas y Certificación ICONTEC. Norma técnica Colombiana 4574 Microbiología de alimentos y alimentos para animales método horizontal para la detección de Salmonella spp. Bogotá, D.C, El Instituto, 2007.

26. Association of Official Agricultural Chemists. Official Methods of Analysis of the AOAC. 12 th ed. Washington, D.C., The Association, 1975.

27. Benzie IFF, Strain JJ. The ferric reducing ability of plasma (FRAP) as a measure of "antioxidant power": the FRAP assay. Anal Biochem. 1996; 239: 70-6.

28. Rojano B, Gaviria C, Gil M, Saez J, Schinella G, Tournier $H$. Actividad antioxidante del isoespintanol en diferentes medios. Vitae 2008; 15: 173-81.

29. Rojano BA, Zapata-Acosta K, Cortés-Correa FB. Capacidad atrapadora de radicales libres de Passiflora mollissima (Kunth) L. H. Bailey (curuba). Revi Cub Plantas Med. 2012;17:408-19.

30. Prior RL, Wu XL, Schaich $K$. Standardizedmethods for the determination of antioxidant capacity and phenolics in foods and dietary supplements. J Agric Food Chem. 2005; 53: 4290-302.

31. Debnath T, Park P-J, DebNath NC, Samad NB, Park HW, Lim BO. Antioxidant activity of Gardenia jasminoides Ellis fruit extracts. Food Chem. 2011; 128: 697-703.

32. Biswas AK, Sahoo J, Chatli MK. A simple UV-Vis spectrophotometric method for determination of $\beta$-carotene content in raw carrot, sweet potato and supplemented chicken meat nuggets. Food Sci Technol 2011; 44: 1809 - 13.

33. Lutz M, Morales D, Sepúlveda S, Alviña M. Evaluación sensorial de preparaciones elaboradas con nuevos alimentos funcionales destinados al adulto mayor. Rev Chil Nutr. 2008; 35: 131-7.

34. Soliva-Fortuny RC, Mart'in-Belloso O. New advances in extending the shelf-life of fresh-cut fruits: a review. Trends Food Sci Technol. 2003; 14: 341-53.

35. Valderrama $P$, Marangoni $F$, Clemente E. Efeito do tratamento térmico sobre a atividade de peroxidade (POD) e polifenoloxidade (PPO) em maçã (Mallus comunis). Ciência Tecnol Alimentos 2001; 21: 321-25.

36. Stafford AE. Mango. HT Chan Jr. ed. Handbook of tropical foods. New York, Marcel Dekker Inc., 1983, p. 399-31.

37. Tomas-Barberan F, Espin J. Phenolic compounds and related enzymes as determinants of quality in fruits and vegetables. J Sci Food Agric. 2001; 81:853-76.

38. Haytowitz DB, Bhagwat S. Database for the Oxygen Radical Absorbance Capacity (ORAC) of Selected Foods, Release 2. Nutrient Data Laboratory. Agriculture Research Service US Department of Agriculture. Washing ton, D.C., The Departament, 2010. 